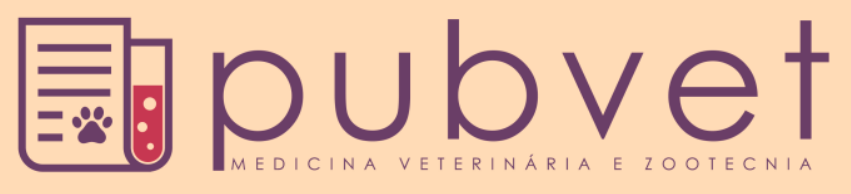

https://doi.org/10.22256/pubvet.v12n2a23.1-8

\title{
Metodologias para a transformação das peles de Linguado, Robalo, Paru e Tilápia em couro
}

\author{
Kátia Kalko Schwarz ${ }^{\ominus}$, Karoline Souza de Mendonça ${ }^{\bullet 2,3}$, Silvia Santos Wakiuchi ${ }^{\bullet}$ 2,3, \\ Jhennifer Caroline Sassamori ${ }^{2,3}$, Gisele Cristina de Júlio Pucci Rebuli ${ }^{3}$
}

1 Professora da Universidade Estadual do Paraná, campus Paranaguá, Departamento de Ciências Biológicas. Paranaguá - PR Brasil.

${ }^{2}$ Aluno(a) do Curso de Ciências Biológicas, da Universidade Estadual do Paraná, campus Paranaguá.

${ }^{3}$ Bolsista Programa Universidade Sem Fronteiras SETI-PR.

*Autora para correspondência, E-mail: katia.kalko@unespar.edu.br.

\begin{abstract}
RESUMO. O objetivo principal deste trabalho foi avaliar a determinação de processos de transformação da pele de peixes em couro, através do uso do tanino vegetal como agente curtente em peles de Linguado (Pleuronectesli neatus) e Robalo flecha (Centropomus undecimalis) e análise de resistência dos couros de Tilápia do Nilo (Oreocchromis niloticus) jovens e adultas e Paru (Chaetodipterus faber). Foram realizados curtimentos, divididos em duas etapas: Processos de Ribeira - curtimento (armazenamento, descarne, remolho, caleiro, desencalagem e píquel) e Processos de Recurtimento - acabamento (neutralização, recurtimento - tingimento, engraxe e secagem). Para as quatro espécies utilizadas durante o experimento, houve redução no uso de ácido fórmico. A proporção inicial era de $1 \%$ diminuindo para $0,5 \%$ na etapa de recurtimento, pois ao entrar em contato direto com as peles, a alta acidificação prejudicou o processo de curtimento e também a diminuição de $2 \%$ da soda barrilha para $1 \%$ durante o processo de caleiro, pois foi observado o rompimento das lamínulas de inserção das escamas, prejudicando o produto final. $\mathrm{O}$ curtimento dos couros de robalos e linguados resultou em um couro duro, sem condições para a realização das análises de resistência mecânicas, necessitando do desenvolvimento de novas metodologias para obtenção de um produto final macio e de fácil manuseio. Os corpos de prova foram feitos para os testes de: tração $\left(\mathrm{N} / \mathrm{mm}^{2}\right)$, alongamento $(\%)$ e rasgamento progressivo $(\mathrm{N} / \mathrm{mm})$, para os couros de tilápia e paru no dinamômetro EMIC ${ }^{\circledR}$ com velocidade de afastamento entre cargas de $100 \pm 20 \mathrm{~mm} / \mathrm{mm}$. Os Couros de tilápias jovens e adultas apresentaram resistência à tração de $63,3 \mathrm{~N} / \mathrm{mm}^{2} \mathrm{e}$ $42,6 \mathrm{~N} / \mathrm{mm}^{2}$, ao alongamento de $80,9 \%$ e $64,3 \%$ e ao rasgamento progressivo de 32,7 $\mathrm{N} / \mathrm{mm}$ e 41,9 N/mm, respectivamente. Comprovando superioridade em relação ao couro de Paru onde apresentou resistência a tração de $56,8 \mathrm{~N} / \mathrm{mm}^{2}$, ao alongamento de 42,5\% e ao rasgamento progressivo de $20,9 \mathrm{~N} / \mathrm{mm}$. Os couros de tilápias jovens e adultas e de Parus de acordo com a ABNT NBR 13525:2016 podem ser empregados para cabedal de moda, e os das tilápias também podem ser utilizados para fins automotivos e moveis.
\end{abstract}

Palavras chave: Curtimento, peixe, processo, produto

\section{Tanning methodologies using skins of righteye Pleuronectes neatus, Centropomus undecimalis, Oreocchromis niloticus and Chaetodipterus faber}

ABSTRACT. This study aimed to evaluate the determination of tanning processes with fish skin, using vegetable tannins as tanning agent in righteye Pleuronectes neatus and Centropomus undecimalis. The study also performed a leather resistance analysis for young and adult Chaetodipterus faber and Chaetodipterus faber Chaetodipterus faber. The 
carried out tanning is divided into two steps: Beamhouse operations - tanning (curing, unhairing, scudding, soaking, liming, deliming and pickling) and finishing operations (neutralising, retanning, drum dyeing, fatliquoring and drying). The use of formic acid, with an initial proportion of $1 \%$, suffered $0.5 \%$ reduction at the retanning stage for the four species in the experiment. The high acidification in direct contact with the skins adversely affected the tanning process, causing also the decrease from $2 \%$ to $1 \%$ of soda ash during the liming process, being noticed the rupture of scales insertion coverslips, damaging the final product. The tanning of Centropomus undecimalis and Pleuronectes neatus resulted in hard leather, which does not provide conditions for the mechanical resistance analysis, thus requiring the development of new methodologies to obtain a soft and easy-to-handle final product. The proof-bodies were made for the following tests: tensile strength $\left(\mathrm{N} / \mathrm{mm}^{2}\right)$, elongation and progressive tearing $(\mathrm{N} / \mathrm{mm})$ for tilapia and Chaetodipterus faber leathers, using the $\mathrm{EMIC}^{\circledR}$ dynamometer at removal speed of $100 \pm 20 \mathrm{~mm} / \mathrm{mm}$. Regarding the mechanical resistance analysis results, the leather of young and adult Oreocchromis niloticus respectively presented values of $63.3 \mathrm{~N} / \mathrm{mm}^{2}$ and 42.58 for tensile strength, $80.9 \%$ and $64.3 \%$ for elongation and $32.7 \mathrm{~N} / \mathrm{mm}$ and $41.9 \mathrm{~N} / \mathrm{mm}$ for progressive tearing, which proves its superiority over the Chaetodipterus faber. This one presented tensile strength of $56.8 \mathrm{~N} / \mathrm{mm}^{2}$ and progressive tearing resistance of $20.9 \mathrm{~N} / \mathrm{mm}$. Young and adult Oreocchromis niloticus and Centropomus undecimalis, according to ABNT NBR 13525:2015, can be used for fashion leather. Oreocchromis niloticus, exclusively, can also provide leather for automotive purposes and furniture.

Keywords: Tanning, fish, process, product

\section{Metodologías para la transformación de pieles de Pleuronectesli neatus, Centropomus undecimalis, Oreocchromis niloticus y Chaetodipterus faber en cuero}

RESUMÉN. Este estudio tuvo como objetivo evaluar la determinación de procesos de transformación de la piel de peces en cuero, utilizando taninos vegetales como agente curtidor en pieles de Pleuronectesli neatus y Centropomus undecimalis. También se realizó análisis de resistencia de los cueros de Oreocchromis niloticus jóvenes y adultos y Chaetodipterus faber. Fueron realizadas curtibres, divididas en dos etapas: Proceso de Ribera - curtido (Almacenamiento, descarne, remojo, encalado, desencalado) y proceso de acabado - Recurtido (neutralización, recurtido, tinte, engrase y secado). Para las cuatro especies utilizadas durante el experimento, hubo reducción en el uso de ácido fórmico. La proporción inicial era de $1 \%$ siendo reducida para $0,5 \%$ en la etapa de recurtido, pues al entrar en contacto directo con las pieles, la elevada acidificación afectó negativamente el proceso de curtimbre e también la disminución del $2 \%$ para el $1 \%$ de soda durante el proceso de curtimbre, notándose la ruptura de las láminas de inserción de las escamas, dañando el producto final. El curtido de los cueros Pleuronectesli neatus y Centropomus undecimalis resultó en un cuero duro, que no proporcionó condiciones para el análisis de resistencia mecánica, lo que requiere del desarrollo de nuevas metodologías para obtener un producto final suave y fácil de manejar. Los cuerpos de prueba fueron hechos para los ensayos de tracción $\left(\mathrm{N} / \mathrm{mm}^{2}\right)$, alargamiento $(\%)$ y desgarramiento progresivo $(\mathrm{N} / \mathrm{mm} 2)$, para cueros de Oreocchromis niloticus y Chaetodipterus faber, utilizando el dinamómetro EMIC® a una velocidad de separación entre cargas de $100 \pm 20 \mathrm{~mm} / \mathrm{mm}$. El cuero de Oreocchromis niloticus jóvenes y adultas presentaron resistencia a la tracción de 63,3 $\mathrm{N} / \mathrm{mm}^{2}$ y $42,6 \mathrm{~N} / \mathrm{mm}^{2}$, al alargamiento $80,9 \%$ y $64,3 \%$ y a la rasgaduras progresivas 32,7 $\mathrm{N} / \mathrm{mm}$ y $41,9 \mathrm{~N} / \mathrm{mm}$, respectivamente. Comprobando superioridad en relación al cuero de Chaetodipterus faber, el cual presentó resistencia a tracción de $56,8 \mathrm{~N} / \mathrm{mm}^{2}$, al alargamiento de $42,5 \%$ y al rasgamiento progresivo de $20,9 \mathrm{~N} / \mathrm{mm}$. Los cueros de Oreocchromis niloticus jóvenes y adultas y de Chaetodipterus faber de acuerdo con la 
ABNT NBR 13525:2016 pueden ser empleados para exclusividad de moda, y los de Oreocchromis niloticus también pueden ser utilizados en automóviles y muebles.

Palabras clave: Curtimbre, peces, proceso, producto

\section{Introdução}

O processamento de peixes, seja na indústria, peixarias ou ainda em mercados municipais, tem gerado uma elevada quantidade de resíduos. Este pode ultrapassar $60 \%$ em relação ao peso total do animal, sendo a pele cerca de 5 a $10 \%$ conforme a espécie e tamanho de peixe (Souza, 2008, Viegas \& Souza, 2011, Franco et al., 2014). Aproveitar a pele, que seria descartada, para transformar em couro tem sido um desafio a ser desenvolvido, para a geração de novas tecnologias de curtimento, bem como na diminuição deste subproduto no ambiente, transformando em eco-produto com valor agregado (Cardoso, 2010, Franco et al., 2014).

O uso de agentes do curtimento também apresenta grande influência no produto final. Atualmente demonstra-se vantagem no uso de taninos vegetais e sintéticos, pois estes são responsáveis por encorpar a espessura do couro, auxiliando na sua resistência ao rasgamento, flexibilidade e maciez, além de ser um produto ecologicamente correto, quando comparado ao cromo (Cardoso, 2010, Eiras et al., 2015).

O processamento de couros de tilápia tem sido estudado constantemente, principalmente pela grande produção e abundância desta espécie de peixe. De acordo com o Ministério do Meio Ambiente (2016) a pesca extrativista do Paru no Brasil foi em 2009 de 270,7 ton/ano, $2010 \mathrm{em}$ torno de 255,9 ton/ano e em 2011 chegou a 258,1 ton ao ano. Como esta espécie é estuarina, tem sido abundante no litoral paranaense, seja pelas condições ambientais e ou ainda pela facilidade de captura nos meses mais quentes do ano, parece ser interessante o aproveitamento desta pele em couro, bem como de linguados e robalos.

Avaliar a resistência mecânica dos couros de peixes, e o correto uso destes produtos beneficiando as comunidades de pescadores, aquicultores e artesãos do litoral paranaense, quanto ao processo de curtimento foi o objetivo principal deste estudo.

\section{Material e Métodos}

Os estudos foram desenvolvidos no Laboratório Multidisciplinar de Estudos Animais da Universidade Estadual do Paraná campus de
Paranaguá, e no Curtume Artesanal Comunitário de Pontal do Paraná/PR-Brasil no PROVOPAR (Programa do Voluntariado Paranaense) Balneário Praia de Leste em Pontal do Paraná - PR.

Neste trabalho foram realizados curtimentos para a transformação da pele de peixe em couro. Para este processo foi curtido a pele de quatro espécies: linguado (Paralichthys patagonicus Jordan, 1889), robalo flecha (Centropomus undecimalis - Bloch, 1792) tilápia do nilo (Oreochromis niloticus - Linnaeus, 1758) jovens $(450 \mathrm{~g})$ e adultas $(800 \mathrm{~g})$, e do paru (Pomacanthus paru - Bloch, 1787) pescados na baía de Paranaguá, e obtidos no mercado Municipal de Peixes de Paranaguá - PR. Foram utilizados cinco quilogramas de peles in natura, de cada tipo de pele analisada.

Para o processo de curtimento das peles, a metodologia empregada foi adaptada de Hoinacki (1989) e Souza (2004) utilizando como agente curtente o tanino vegetal de acácia no curtimento e no recurtimento o tanino sintético, em um fulão de madeira com rotação de $14 \mathrm{rpm}$. As etapas para o processo de curtimento, dosagens e tempos de cada fase do processo de transformação das peles de peixes em couro estão representadas na Tabela 1 .

$\mathrm{Na}$ realização desse trabalho, buscou-se o emprego de tecnologias simples e possíveis de serem executadas, em curto período de tempo, com auxílio de equipamentos e recursos humanos disponíveis, assim como a análise dos couros curtidos por testes físicos - mecânicos.

Para as espécies tilápia do nilo e Paru houve uma redução na proporção de soda barrilha, durante a etapa de Caleiro. No processo padrão utilizou-se $2 \%$ de soda barrilha sendo necessária a redução para $1 \%$ durante $\mathrm{o}$ processo de curtimento. Também houve a redução no uso de ácido fórmico, onde a proporção inicial era de $1 \%$ diminuindo para $0,5 \%$ durante as etapas de Píquel, Recurtimento/Tingimento e Engraxe (Tabela 2).

Para as espécies linguado e robalo flecha também houve a redução no uso de soda barrilha, diminuindo de $2 \%$ inicialmente para $0,5 \%$ ao final do processo. Também houve a redução no uso de ácido fórmico, onde a proporção inicial era de $1 \%$ 
diminuindo para $0,5 \%$ durante as etapas de Píquel, Recurtimento/Tingimento e Engraxe (Tabela 3).

Os testes físicos - mecânicos foram realizados pelo Instituto de Tecnologia do Paraná - TECPAR de Maringá-PR. Os corpos de provas foram retirados dos couros para determinação dos testes de tração - N/mm², alongamento (\%) (ABNT NBR 11041, 1997) e de rasgamento progressivo$\mathrm{N} / \mathrm{mm}$. Foram utilizados 10 couros retirados aletoriamente do montante curtido de cada tipo de pele a ser analisada.

Tabela 1. Mensuração de reagentes de duração de cada etapa do processo de curtimento, metodologia inicial

\begin{tabular}{|c|c|c|}
\hline Etapas & Reagentes & Observação \\
\hline Remolho & $\begin{array}{l}200 \% \text { de água } \\
2 \% \text { tensoativo }\end{array}$ & 1 hora - Esgotar ${ }^{1}$ \\
\hline Caleiro & $\begin{array}{l}200 \% \text { de água } \\
3 \% \text { Hidróxido de Cálcio } \\
2 \% \text { Soda barrilha } \\
1 \% \text { tensoativo }\end{array}$ & 2 horas - Esgotar \\
\hline Desencalagem & $\begin{array}{l}\text { 200\% de água } \\
\text { 0,5\% Desencalante }\end{array}$ & 30 minutos - Esgotar \\
\hline Purga & $\begin{array}{l}200 \% \text { de água } \\
1 \% \text { Enzima proteolítica } \\
1 \% \text { Tensoativo } \\
0,5 \% \text { Desencalante }\end{array}$ & 1 hora - Esgotar \\
\hline Desengraxe & $\begin{array}{l}200 \% \text { de água } \\
2 \% \text { Querosene } \\
1 \% \text { Tensoativo }\end{array}$ & 1 hora - Esgotar \\
\hline Píquel & $\begin{array}{l}200 \% \text { de água } \\
6 \% \text { Sal } \\
1 \% \text { Ácido Fórmico } \\
2 \% \text { Alvejante } \\
10 \% \text { Tanino vegetal }\end{array}$ & $\begin{array}{l}30 \text { min. } 30 \text { min., } 3 \text { X10 min. }{ }^{2} \\
30 \text { min. } \\
2 \text { horas, descanso de } 12 \text { horas das peles } \\
\text { submersas nesta solução }\end{array}$ \\
\hline Neutralização & $\begin{array}{l}200 \% \text { de água } \\
0,5 \% \text { Bicarbonato de Sódio }\end{array}$ & 30 minutos - Esgotar \\
\hline Recurtimento/Tingimento & $\begin{array}{l}200 \% \text { água } \\
1 \% \text { Tanino sintético } \\
3 \% \text { Tanino vegetal } \\
1 \% \text { Anilina para couro } \\
3 \% \text { Tanino vegetal } \\
1 \% \text { Anilina para couro } \\
1 \% \text { Ácido fórmico }\end{array}$ & $\begin{array}{l}30 \text { minutos } \\
30 \text { minutos } \\
30 \text { minutos, } 3 \text { X10 minutos - Esgotar }\end{array}$ \\
\hline Engraxe & $\begin{array}{l}200 \% \text { água a } 60{ }^{\circ} \mathrm{C} \\
4 \% \text { Óleo sulfitado } \\
4 \% \text { Óleo sulfatado } \\
2 \% \text { Catalix } \\
1 \% \text { Ácido fórmico }\end{array}$ & $\begin{array}{l}1 \text { hora } \\
30 \text { minutos, } 3 \text { X10 minutos }- \text { Esgotar }\end{array}$ \\
\hline
\end{tabular}

${ }^{1}$ Esgotar: Após cada processo a solução é esgotada do fulão e este é lavado para uso da próxima etapa. As peles são retiradas, lavadas em água corrente, retirando o excesso de água, e colocadas novamente no fulão, em nova solução, conforme etapas do processo. ${ }^{2} \mathrm{~A}$ cada 10 minutos 1/3 de ácido fórmico, diluído em $100 \mathrm{ml}$ de água, foi adicionado na solução, com o fulão em rotação.

No Tecpar unidade de Maringá, os couros foram levados para um ambiente climatizado em torno de $23^{\circ} \mathrm{C}$ e umidade relativa do ar de $50 \%$, por 24 horas (ABNT - NBR 10455, 1988). Para os testes de resistência foi utilizado o dinamômetro da marca EMIC®, com velocidade de afastamento entre cargas de $100 \pm 20 \mathrm{~mm} / \mathrm{mm}$.

O laboratório forneceu um laudo dos resultados obtidos com a média total, de cada tipo de couro analisado, ou seja, um único valor para tilápia jovem, outro para a adulta, assim como para o Paru.

Os resultados obtidos foram comparados com a ABNT NBR 13525:2016 - Ensaios físicos e químicos em couro - valores de orientação para aceitação de couros. Esta norma estabeleceu parâmetros de acordo com os resultados dos testes de resistência a aplicação do uso destes couros. 
Tabela 2. Alterações no processo de curtimento para as espécies de tilápia do nilo (Oreochromis niloticus) e Paru (Pomacanthus paru)

\begin{tabular}{|c|c|c|}
\hline Etapa & Processo inicial & Processo final \\
\hline Caleiro & $\begin{array}{l}200 \% \text { água } \\
3 \% \text { Hidróxido de cálcio } \\
2 \% \text { Soda barrilha } \\
1 \% \text { Tensoativo }\end{array}$ & $\begin{array}{l}200 \% \text { água } \\
3 \% \text { Hidróxido de cálcio } \\
1 \% \text { Soda barrilha } \\
1 \% \text { Tensoativo }\end{array}$ \\
\hline Píquel & $\begin{array}{l}\text { 6\% Sal } \\
1 \% \text { Ácido Fórmico } \\
2 \% \text { Alvejante } \\
10 \% \text { Tanino vegetal }\end{array}$ & $\begin{array}{l}\text { 6\% Sal } \\
0,5 \% \text { Ácido Fórmico } \\
2 \% \text { Alvejante } \\
10 \% \text { Tanino vegetal }\end{array}$ \\
\hline Recurtimento/Tingimento & $\begin{array}{l}\text { 100\% água } \\
1 \% \text { Tanino sintético } \\
3 \% \text { Tanino vegetal } \\
1 \% \text { Anilina para couro } \\
3 \% \text { Tanino vegetal } \\
1 \% \text { Anilina para couro } \\
1 \% \text { Ácido fórmico }(3 \times 10)\end{array}$ & $\begin{array}{l}\text { 100\% água } \\
1 \% \text { Tanino sintético } \\
3 \% \text { Tanino vegetal } \\
1 \% \text { Anilina para couro } \\
3 \% \text { Tanino vegetal } \\
1 \% \text { Anilina para couro } \\
0,5 \% \text { Ácido fórmico }(3 \times 10)\end{array}$ \\
\hline Engraxe & $\begin{array}{l}100 \% \text { água a } 60{ }^{\circ} \mathrm{C} \\
4 \% \text { Óleo sulfitado } \\
4 \% \text { Óleo sulfatado } \\
2 \% \text { Catalix } ® \text { (agente de toque) } \\
1 \% \text { Ácido fórmico (3X10) }\end{array}$ & $\begin{array}{l}100 \% \text { água a } 60{ }^{\circ} \mathrm{C} \\
4 \% \text { Óleo sulfitado } \\
4 \% \text { Óleo sulfatado } \\
2 \% \text { Catalix }{ }^{\circledR} \text { (agente de toque) } \\
0,5 \% \text { Ácido fórmico }(3 \times 10)\end{array}$ \\
\hline
\end{tabular}

Tabela 3. Alterações no processo de curtimento para as espécies de linguado (Pleuronectesli neatus) e robalo flecha (Centropomus undecimalis)

\begin{tabular}{lll}
\hline Etapa & Processo inicial & Processo final \\
\hline Caleiro & $200 \%$ água & $200 \%$ água \\
& $3 \%$ Hidróxido de cálcio & $3 \%$ Hidróxido de cálcio \\
& $2 \%$ Soda barrilha & $0,5 \%$ Soda barrilha \\
& $1 \%$ Tensoativo & $1 \%$ Tensoativo \\
Píquel & $6 \%$ Sal & $6 \%$ Sal \\
& $1 \%$ Ácido Fórmico & $0,5 \%$ Ácido Fórmico \\
& $2 \%$ Alvejante & $2 \%$ Alvejante \\
& $10 \%$ Tanino vegetal & $10 \%$ Tanino vegetal \\
Recurtimento/Tingimento & $100 \%$ água & $100 \%$ água \\
& $1 \%$ Tanino sintético & $1 \%$ Tanino sintético \\
& $3 \%$ Tanino vegetal & $3 \%$ Tanino vegetal \\
& $1 \%$ Anilina para couro & $1 \%$ Anilina para couro \\
& $3 \%$ Tanino vegetal & $3 \%$ Tanino vegetal \\
& $1 \%$ Anilina para couro & $1 \%$ Anilina para couro \\
& $1 \%$ Ácido fórmico $(3 \mathrm{X} 10)$ & $0,5 \%$ Ácido fórmico $(3 \mathrm{X} 10)$ \\
& $100 \%$ água a $60{ }^{\circ} \mathrm{C}$ & $100 \%$ água a $60{ }^{\circ} \mathrm{C}$ \\
Engraxe & $4 \%$ Óleo sulfitado & $4 \%$ Óleo sulfitado \\
& $4 \%$ Óleo sulfatado & $4 \%$ Óleo sulfatado \\
& $2 \%$ Catalix® $($ agente de toque) & $2 \%$ Catalix® (agente de toque) \\
& $1 \%$ Ácido fórmico $(3 \mathrm{X} 10)$ & $0,5 \%$ Ácido fórmico $(3 \mathrm{X} 10)$ \\
\hline
\end{tabular}

\section{Resultados e Discussão}

Os couros de robalos e linguados ficaram duros e ressecados, a tal pondo de inviabilizar o uso para as análises de resistência mecânica. De acordo com Yoshida et al. (2016) couros de peixes muito duros podem ocorrer por diversos fatores, entre valores de $\mathrm{pH}$ baixo, pode causar perda de água pelo tecido e consequente desnaturação das proteínas (colágeno) presente nas peles de peixe, podendo as tornar mais duras e/ou ressecadas, dificultando ainda a penetração de óleos no engraxe. De fato, pode ter ocorrido o problema do $\mathrm{pH}$ baixo, mas também o tempo de caleiro pode ter sido insuficiente para a abertura das fibras, dosagem de alguns agentes químicos de curtimento durante o processo, tempo de exposição da pele in natura frente a cada reagente 
curtente e a falta da repetição de algumas fases iniciais do processo (Quaresma \& Moura, 2016, Yoshida et al., 2016) devem ser estudadas futuramente para a obtenção de couros de robalos e linguados.

Com relação a transformação da pele de tilápias jovens e adultas e de Parus, o couro obtido foi macio, porém para as tilápias adultas apresentou um pouco mais entumecido. Para cada espécie de peixe, de acordo com a sua idade, sexo, alimentação e espécie a pele pode apresentar características diferentes, sendo que para cada espécie de peixe e tamanho uma metodologia própria deve ser aplicada (Souza, 2004, Souza \& Silva, 2005, Souza et al., 2006, Vieira et al., 2008).

$\mathrm{Na}$ Tabela 4, constam os resultados da determinação de resistência à tração $\left(\mathrm{N} / \mathrm{mm}^{2}\right)$, ao alongamento (\%) e ao rasgamento progressivo $(\mathrm{N} / \mathrm{mm})$ para as tilápias e paru. Para os testes físicos - mecânicos utilizou-se a análise de 10 couros de cada espécie estudada, sendo feita a média para cada um dos resultados obtidos.

Tabela 4. Média dos testes de resistência do couro de tilápia do Nilo (jovens e adultas) e Paru, submetidas à técnica de curtimento de taninos vegetais

\begin{tabular}{llll}
\hline Resistência mecânica & Tilápia do Nilo (jovem) & Tilápia do Nilo (adulta) & Paru \\
\hline Tração (N/mm²) & $63,30 \mathrm{~N} / \mathrm{mm}^{2}$ & $42,58 \mathrm{~N} / \mathrm{mm}^{2}$ & $56,84 \mathrm{~N} / \mathrm{mm}^{2}$ \\
Alongamento (\%) & $80,88 \%$ & $64,30 \%$ & $42,48 \%$ \\
Rasgamento Progressivo (N/mm) & $32,66 \mathrm{~N} / \mathrm{mm}$ & $41,87 \mathrm{~N} / \mathrm{mm}$ & $20,89 \mathrm{~N} / \mathrm{mm}$ \\
\hline
\end{tabular}

As peles de tilápia do nilo jovem e adulta, curtida com taninos vegetais, apresentaram resistência à tração de $63,3 \mathrm{~N} / \mathrm{mm}^{2}$ e $42,6 \mathrm{~N} / \mathrm{mm}^{2}$, ao alongamento de $80,9 \%$ e $64,3 \%$ e ao rasgamento progressivo de $32,7 \mathrm{~N} / \mathrm{mm}$ e 41,9 $\mathrm{N} / \mathrm{mm}$ respectivamente; comprovando superioridade em relação ao couro de Paru onde apresentou resistência a tração de $56,8 \mathrm{~N} / \mathrm{mm}^{2}$, ao alongamento de $42,5 \%$ e ao rasgamento progressivo de $20,9 \mathrm{~N} / \mathrm{mm}$.

A diferença nos resultados de resistência das peles de tilápia e paru foi devido as características da epiderme. De acordo com Souza (2004) conforme a espécie, tamanho e características da epiderme, os resultados de resistência dos couros podem variar. As peles de tilápia jovens eram de peixes abatidos com $350 \mathrm{~g}$, as de adultas de $800 \mathrm{~g}$, e os Parus cerca de 1,2 kg.

A pele de Paru in natura apresentou uma característica interessante, escamas pequenas, pele extremamente gordurosa, apresentado uma camada lipídica entre a derme de coloração amarelada e fétida. Todavia, o fator do odor da gordura da pele do Paru, não interferiu no resultado final do couro curtido, pois pode ter sido retirado durante a etapa de desengraxe com o uso de querosene e na de piquel com o alvejante, produtos estes que tem a função de desodorização.

O curtimento com tanino vegetal e sintético e/ou com a combinação dos taninos vegetais e sintéticos pode ter influenciado na resistência dos couros de tilápia. As peles de tilápia, quando curtidas e recurtidas apenas com tanino sintético, independentemente da concentração (10 ou 12\%), apresentaram pior resistência à tração. Quando comparados com os que receberam $10 \%$ de taninos (vegetal e a combinação dos taninos), esses couros apresentaram melhores resultados na resistência (Vieira et al., 2008).

Para Souza et al. (2006) em análise de couro de tilápia do nilo, os couros curtidos com sais de cromo apresentaram resistência a tração de 12,2 $\mathrm{N} / \mathrm{mm}^{2}$, ao alongamento de $93,08 \%$ e ao rasgamento progressivo de $25,5 \mathrm{~N} / \mathrm{mm}$, enquanto, nesse experimento, a resistência medida nas peles de tilápia foi superior para tração e rasgamento progressivo em tilápias jovens. O uso do tanino vegetal de Acássia pode ter influenciado neste resultado, como já observado nos estudos de Vieira et al. (2008). Estes autores concluíram em seus estudos, que os couros de tilápia do nilo curtidos com $10 \%$ de tanino vegetal apresentou resistência a tração de $70,2 \mathrm{~N} / \mathrm{mm}^{2}$ e a combinação dos taninos vegetal e sintético variaram entre 87,5 $\mathrm{N} / \mathrm{mm}^{2} \mathrm{e} \quad 89,6 \mathrm{~N} / \mathrm{mm}^{2}$, sendo esses valores superiores aos observados neste experimento.

Segundo Souza et al. (2006) a resistência ao rasgamento progressivo para o couro da tilápia do Nilo com curtimento bioleather $^{\circledR}$ foi de 13,1 $\mathrm{N} / \mathrm{mm}$. Neste experimento, o rasgamento progressivo foi de $32,7 \mathrm{~N} / \mathrm{mm}$ para jovens e 41,87 $\mathrm{N} / \mathrm{mm}$, para adultas, sendo superior com o uso de taninos vegetais. Machado (2001) analisando o couro no sentido longitudinal de Piavuçu, 
Piraputanga e Pacu prata observou que porcentagem de alongamento ou elongação até a ruptura não diferiu para Piavuçu $(52,8 \%)$ e Piraputanga $(60,5 \%)$. O resultado obtido neste experimento para o Paru foi semelhante ao observado por Machado (2001) para o couro do Piavuçu.

Para Hoinacki (1989) os valores de referência da resistência do couro bovino curtido ao cromo para elongação ou alongamento até a ruptura, são no mínimo 60\%; para a tração, no mínimo 9,8 $\mathrm{N} / \mathrm{mm}^{2}$; para o rasgamento progressivo, 14,7 $\mathrm{N} / \mathrm{mm}$, sendo os valores observados durante o experimento adequados para a utilização desses couros na indústria de calçados e vestuários.

Os resultados obtidos neste estudo, com a metodologia empregada de curtimento sugerem que os couros de tilápias jovens e adultas e de Parus de acordo com a ABNT NBR 13525:2016 podem ser empregados para cabedal de moda e os das tilápias também podem ser utilizados para fins automotivos e moveis.

\section{Conclusões}

A metodologia padrão utilizada durante o processo de curtimento mostrou-se insuficiente para robalos e linguados, sendo necessárias adaptações em quantidade de reagentes em determinadas etapas do curtimento, bem como da repetição de algumas fases. As peles de tilápia do Nilo, tanto jovens quanto as adultas, apresentaram melhor desempenho durante o processo de curtimento, quando comparadas com os couros de Paru, e de resistência mecânica também.

Novas pesquisas devem ser realizadas para o aproveitamento das peles de peixes marinhos e dulcícolas, encontrados em grande quantidade da região do litoral paranaense. Assim, criando alternativas de geração de trabalho e renda para as comunidades de pescadores, aquicultores e artesãos da região. Esta técnica pode ser ainda reestudada, com o propósito de obtenção de um produto final de melhor qualidade, maciez e de amplo uso na indústria de artefatos de couro.

\section{Agradecimentos}

Unespar, campus Paranaguá, Fundação Araucária, Programa Universidade Sem Fronteiras e SETI/UGF/PR (Secretaria de Estado de Ciência e Tecnologia do Paraná/Unidade Gestora do Fundo Paraná).

\section{Referências Bibliográficas}

Associação brasileira de normas técnicas - ABNT. NBR 10455: climatização de materiais usados na fabricação de calçados e correlatos. Rio de Janeiro: p.1-2, 1988.

Associação brasileira de normas técnicas - ABNT. NBR 11041: couros - determinação da resistência à tração e alongamento. Rio de Janeiro: p.1-5, 1997.

Associação brasileira de normas técnicar-ABNT. 13525: couro -Ensaios físicos e químicos em couro - Valores orientativos para aceitação de couros. Rio de Janeiro, 2016.p.1-9.

Cardoso, J. 2010. O design industrial como ferramenta para a sustentabilidade: estudo de caso do couro de peixe. Revista Espaço Acadêmico, 10, 110-117.

Eiras, B. J. C. F., Medeiros Júnior, E. F. \& Alves, M. M. 2015. Desenvolvimento de método artesanal de curtimento da pele da pescada amarela (Cynoscion acoupa), e sua difusão por meio de oficina a uma comunidade no município de Bragança, PA, Brasil. Semina: Ciências Agrárias, 36, 1123-1134.

Franco, M. L. R. S., Prado, M., Fernandes, V. R. T., Delbem, Á. C. B., Lara, J. A. F., Bielawski, K., Gasparino, E. \& Del Vesco, A. P. 2014. Pele de Surubim: morfologia e resistência do couro com adição de óleo no engraxe. Acta Tecnológica, 9, 1-8.

Hoinacki, E. 1989. Peles e couros; origens, defeitos, industrialização. SENAI, Porto Alegre, Rio Grande so Sul.

Machado, S. D. 2001. Aproveitamento e tecnologia do curtimento de pele de peixe. Universidade para o Desenvolvimento do Estado e da Região do Pantanal, Campo Grande, Mato Grosso do Sul.

Ministério do meio ambiente- secretaria de biodiversidade e florestas. 2016. $5^{\circ}$ Relatório Nacional para a Convenção sobre Diversidade Biológica. Brasília.

Quaresma, D. M. M. \& Moura, H. T. 2016. Design para a sustentabilidade ampla de sistemas produto-serviço: estudo de caso de empresa de design de acessórios de moda em couro. Estudos em Design, 24, 66-91.

Souza, M. L. R. 2004. Tecnologia para processamento das peles de peixe. Fundamentum, 1, 14-55. 
Souza, M. L. R. 2008. Tecnologia para processamento das peles de peixe. Fundamentum, 1, 14-55.

Souza, M. L. R., Casaca, J. d. M., Nakaghi, L. S. O., Franco, N. d. P., Silva, L. O., Dourado, D. M. \& Viegas, E. M. M. 2006. Efeito da técnica de curtimento e do método utilizado para remoção da pele da tilápia-do-nilo sobre as características de resistência do couro. Revista Brasileira de Zootecnia, 35, 1273-1280.

Souza, M. L. R. \& Silva, L. O. 2005. Efeito de técnicas de recurtimento sobre a resistência do couro da tilápia do Nilo (Oreochromis niloticus L.). Acta Scientiarum. Animal Sciences, 27, 535-540.

Viegas, E. M. M. \& Souza, M. L. R. 2011. Técnicas de Processamento de Peixes. Centro de Produções Técnicas, Viçosa, Minas Gerais.
Vieira, A. M., Kachba, Y. R., Franco, M. L. R. S., Oliveira, K. F., Godoy, L. C. \& Gasparino, E. 2008. Curtimento de peles de peixe com taninos vegetal e sintético. Acta Scientiarum. Animal Sciences, 30, 359-363.

Yoshida, G. M., Kunita, N. M., Souza, M. L. R. \& Gasparino, E. 2016. Análises mecânicas e físico-químicas de couros de tilápia, cachara e salmão. Archivos de Zootecnia, 65, 349-355.

Article History:

Received 20 September 2017

Accepted 23 October 2017

Available online 28 December 2017

License information: This is an open-access article distributed under the terms of the Creative Commons Attribution License 4.0, which permits unrestricted use, distribution, and reproduction in any medium, provided the original work is properly cited. 